\title{
Non-linear EEG synchronization during observation and execution of simple and complex sequential Finger movements
}

\author{
Claire Calmels $\cdot$ Magaly Hars $\cdot$ Paul Holmes $\cdot$ \\ Gilbert Jarry $\cdot$ Cornelis J. Stam
}

\begin{abstract}
The main aim of this study was to examine the temporal aspects of neuronal changes during the observation and execution of simple and complex tasks to gain a greater understanding of the mirror neuron system's involvement in complex motor tasks. Eleven right-handed subjects observed simple and complex Wnger movement sequences. Electroencephalograms were recorded from 19 electrodes. Activity was considered in four frequency bands $(8-10,10-13,13-20$, and $20-30 \mathrm{~Hz})$ using a new measure, synchronization likelihood. The results show that motor tasks of diVerent levels of complexity did not have a signiWcant inXuence on cortical synchronization. The results also provide additional indirect evidence for mirror neuron activity associated with intransitive tasks. Data are discussed in the light of recent Wndings from the cognitive and behavioral neuroscience literature.
\end{abstract}

C. Calmels (\&) - M. Hars

Département des Sciences du Sport,

Institut National du Sport et de l'Education Physique,

11 Avenue du Tremblay, 75012 Paris, France

e-mail: claire.calmels@insep.fr

M. Hars

Université de Reims Champagne-Ardenne, Reims, France

P. Holmes

Research Institute for Health and Social Change,

Manchester Metropolitan University, Manchester, UK

G. Jarry

Hôpital Henri Mondor, Créteil, France

C. J. Stam

Department of Clinical Neurophysiology,

VU University Medical Centre, Amsterdam, The Netherlands
Keywords Action observation - Synchronization · Mirror neurons · Movement complexity · Electroencephalography

\section{Introduction}

Mirror neurons (MN) were Wrst discovered in the ventral premotor cortex of the macaque monkey with single neuron recording (Di Pellegrino et al. 1992; Gallese et al. 1996; Rizzolatti et al. 1988). These visuomotor neurons show special characteristics; they Wre when the monkey executes a goal-directed hand movement and also when it observes this same action executed by another monkey or by a human.

Evidence for the existence of similar neurons in humans has been provided by researchers who have used electroencephalographic (EEG) techniques (e.g., Cochin et al. 1999; Muthukumaraswamy and Johnson 2004; Muthukumaraswamy et al. 2004) and brain imaging techniques (e.g., Buccino et al. 2001; Grèzes et al. 2003; Iacoboni et al. 1999). EEG research has also provided indirect evidence for the existence of MN in humans. As early as 1954, Gastaut and Bert examined the mu rhythm, an $8-13 \mathrm{~Hz}$ rhythm generated in the sensorimotor cortex. It is known to be suppressed or blocked by the execution of actions and somatosensory stimulations. However, of interest here, Gastaut and Bert (1954) also showed that the mu rhythm was blocked during observation of actions performed by conspeciWcs. This result has been corroborated by more recent Wndings (Cochin et al. 1998, 1999; Lepage and Théoret, 2006; Muthukumaraswamy and Johnson 2004; Muthukumaraswamy et al. 2004). For example, Cochin et al. (1999) found that in the 7.5-10.5 Hz frequency band and for the electrode sites F7, T5, C3, P3, F8, F4, T6, C4, 
and P4 logarithmical spectral power values during the resting condition were signiWcantly higher than the values obtained during the observation and execution of thumb and index Wnger opposition movements. This mu modulation during the observation of movement led some researchers (i.e., Oberman et al. 2007; Pineda 2005; Ulloa and Pineda 2007) to propose that this suppression could be used as a useful marker of MN system activity (see Pineda 2005 for more details). Brain imaging techniques have also provided evidence in favor of the existence of $\mathrm{MN}$ in humans. In contrast to EEG, the greater spatial resolution of brain imaging techniques oVers more information concerning the localization of areas involved during the observation and execution of actions. Areas which form the core of the human MN system are located in the frontal and parietal areas and comprise the rostral part of the inferior parietal lobule, the lower part of the precentral gyrus, and the posterior part of the inferior frontal gyrus (Rizzolatti and Craighero 2004).

The existence of the MN system in humans is now widely recognized. While many of its characteristics remain unknown, attempts to explore MN system characteristics have increased dramatically in the past 10 years. In humans, the system acts diVerently depending upon the forms of observed motor behaviors. For example, the MN system is activated while observing non-object-related actions, so-called intransitive actions (Cochin et al. 1999; Iacoboni et al. 1999; Patuzzo et al. 2003). Further, the MN system shows less activity during observation of objectless actions, where movements are mimicked, compared to the identical movement that includes the object of interest (Buccino et al. 2001; Muthukumaraswamy and Johnson 2004; Muthukumaraswamy et al. 2004). However, two groups of researchers have found diVerent results (Jackson et al. 2006; Jonas et al. 2007). They have reported no involvement of the MN system during the observation of intransitive actions. More speciWcally, they observed no involvement of ventral premotor cortex or inferior frontal gyrus during the observation of vertical, horizontal, and rotational movements of the hand and foot (Jackson et al. 2006).

Movement complexity has also been suggested to inXuence the involvement of the MN system. Although not systematically investigated, only Molnar-Szakacs et al. (2006), using functional magnetic resonance imaging (fMRI), have examined the role of the fronto-parietal MN system. In this study, individuals observed manual object manipulation sequences of diVerent levels of complexity with serrated cups and stacking rings. The authors reported the involvement of the parietal and frontal cortices and higher visual area during the observation of all manipulation sequences. Two aspects of complexity were included in the experimental design: (1) the cognitive hierarchical complexity, related to the complexity of the strategy used and action structure and (2) the motoric complexity related to the motoric manipulation of objects. The authors found that the MN activity was modulated according to the perceived motoric complexity of the action (i.e., the skill required to perform it) but not its cognitive complexity. They concluded that the diVerent object combination strategies were not suitable for adults since the most complex strategy is acquired in childhood at around 3 years. They suggested that future research should examine the involvement of the MN system in more complex action sequences.

Brain mechanisms supporting the execution of simple and complex motor tasks have been broadly investigated using EEG, magnetoencephalography (MEG) (e.g., Leistner et al. 2006; Pulvermuller et al. 1995) and imaging techniques (e.g., Catalan et al. 1998; Dassonville et al. 1998; Hummel et al. 2003; Verstynen et al. 2005). A number of studies have examined Wnger movements where the complexity of such movements can be deWned by factors, such as speed and accuracy (e.g., Dhamala et al. 2003), sequence length (e.g., Catalan et al. 1998; Manganotti et al. 1998; Sadato et al. 1996a), and diYculty of transition between subsequent Wnger movements (e.g., Chen et al. 1997; Hummel et al. 2003). Generally, most of the studies have demonstrated increased brain activity for complex compared to simple movements. For example, Dassonville et al. (1998) used fMRI to investigate cortical motor activity as subjects performed predictable and unpredictable Wnger movement sequences as quickly as possible. The predictable movement sequence was deWned as simple and the unpredictable as complex. Complex movements showed more activity in premotor, cingulate, SMA, pre-SMA, and superior parietal lobule areas compared to simple movements. More speciWcally, EEG studies have shown that the pattern of brain oscillatory activity varies according to the degree of movement complexity (e.g., Hummel et al. 2003; Kitamura et al. 1993; Manganotti et al. 1998; Pulvermuller et al. 1995). Manganotti et al. (1998) used spectral power and coherence to analyze data collected as right-handed subjects performed four diVerent Wnger apposition tasks at $2 \mathrm{~Hz}$. In the 8-12 and $13-20 \mathrm{~Hz}$ frequency bands, spectral power over the bilateral sensorimotor areas decreased which indicated that processing of the motor system was greater as the task became more complex. High levels of coherence were observed between primary motor cortex, premotor cortex, and the SMA during complex tasks. In contrast, lower levels of coherence were revealed during simple tasks. In addition, Pulvermuller et al. (1995) in the $8-12 \mathrm{~Hz}$ frequency band and using spectral analysis found a contralateral activation close to electrode sites $\mathrm{C} 3$ or $\mathrm{C} 4$ in a simple Wnger tapping task, and a bilateral activation over the motor cortices in a Luria task which is a complex Wnger apposition task. Therefore, simple Wnger movements tended to recruit 
unilateral brain areas whereas, more complex movements showed activity in bilateral regions. These Wndings support the idea of transcortical cell assemblies (Pulvermüller and Mohr 1996) which proposes that "only entities with a certain degree of complexity are represented in transcortical assemblies (i.e., large and strong connected neuron populations), while simple representations are organized as local clusters of neurons" (Pulvermüller and Mohr 1996, pp. 562). Dhamala et al. (2003) have also suggested that as complexity increases, additional eVort is required; the eVort being responsible for the ipsilateral motorcortical activity. The relationship may not be clear as these data propose since Chen et al. (1997) and Dassonville et al. (1998) have shown the existence of ipsilateral activity during simple movement sequences, although ipsilateral activity was still greater for complex movement sequences.

The EEG techniques discussed above provide only a linear analysis of the data. One of the concerns with these approaches is that the analysis presumes that the EEG signal is stationary or varies very slowly (Muthuswamy and Thakor 1998). Since the EEG signal is known to be "noisy", pseudo-stochastic, time-varying, non-stationary, and non-linear (Friston 2000; Palus 1996; Stam et al. 1999; Thakor and Tong 2004), linear analysis methods have been argued to be inappropriate for characterizing non-stationary data with rapidly changing interdependencies. Further, they do not identify the non-linear interdependencies in the underlying dynamical system (Stam et al. 2002a; Stam and van Dijk 2002). More speciWcally, spectral analysis technique is the most commonly used method to investigate the MN system in EEG paradigms (e.g., Cochin et al. 1999; Lepage and Théoret 2006). This method has limitations since it has been argued to reveal only part of the relevant information in EEG. It is only as an index of local cortical engagement (Stam et al. 2002b). To address these concerns, Stam and van Dijk (2002) have provided a non-linear index of synchronization in neuronal signaling between distant neuronal populations. Their method estimates the dynamical interdependencies between a time series (e.g., an EEG channel) and one or more other time series (Stam et al. 2002a, b; Stam and van Dijk 2002). Their index, synchronization likelihood (SL), is a marker that describes how strongly a channel is synchronized to all the other channels. Values of SL range from 1 to 0 . Scores at or near 1 indicate maximal synchronization. For purely random correlations, SL tends to 0. Details regarding the SL technique can be found in Montez et al. (2006). For example, Micheloyannis et al. $(2003,2005)$ have also used this non-linear algorithm to examine EEG and (cognitive) task complexity. They showed that SL was able to discriminate cognitive tasks at diVerent complexities. For tasks requiring complex visuosemantic decision, SL between the temporal sites and parieto-occipital sites compared to the remaining recording sites was highest in gamma and lowest in alpha bands. With reduced task complexity, the observed trend was reversed.

With the exception of the work conducted by MolnarSzakacs et al. (2006), the extent of the MN system's involvement in complex motor tasks has not been investigated. Therefore, examining the temporal aspects of neuronal activity changes during the observation and execution of simple and complex motor tasks requires further study. Therefore, the present study considered complex action sequences in which the level of motoric complexity was similar between the simple and complex actions. The level of complexity was not related to the dexterous manipulation of objects. Consequently, sequential Wnger movements with similar sequence length were chosen for this study with movement complexity being deWned by the order in which the Wngers should be moved; a cognitive complexity. In the simple movement condition, adjacent Wngers were moved in one direction. For complex movements, Wngers were required to move in a predetermined order. To allow for the examination of linear and non-linear changes in coupling between diVerent brain areas, the SL measure (Stam and van Dijk 2002) was used.

It was hypothesized that the brain oscillations underlying the observation of sequential Wnger movements would show similarities to those identiWed in the physical execution of the same movement in areas known to possess mirror properties and in frequency bands lying within a range of $8-30 \mathrm{~Hz}$. In view of the literature results related to brain mechanisms and task complexity, it was also expected that complex movements, argued to include greater cognitive demands, would elicit greater interregional synchronization than simple movements under both the observation and execution conditions.

\section{Method}

\section{Subjects}

Eleven right-handed subjects (eight women and three men; mean age $=23.3, \mathrm{SD}=4.5$ ) were included in the study. The subjects were assessed as right-handed by the Edinburgh Handedness Inventory (OldWeld 1971). All participants were scored as strongly right-handed and did not report the presence of any neurological or psychiatric disorders. All subjects provided written informed consent and were kept blind to the goals of the study. The study was approved by the local institutional ethics committee.

Task

Subjects were required to perform two sequential Wnger movements of diVerent complexity (i.e., a simple movement 
and a complex movement) with their right hand. This choice was made to avoid inter-task priming eVects (Solodkin et al. 2001) and to prevent a gradual reduction in cortical activity due to repeated exposure to a same stimulus (Dassonville et al. 1998; Wu et al. 2004). The movements consisted of touching the tip of the right thumb with the tip of the other right hand Wngers while the hand and forearm remained in a supinated position supported on a pillow. A simple or complex sequential Wnger movement comprised eight Wnger contacts performed at a rate of $2 \mathrm{~Hz}$ controlled by a metronome. The duration of the movement was $4 \mathrm{~s}$. Complexity referred to a succession of eight pre-determined, random, and non-repeated contact performed consecutively. The sequence of the simple sequential movement was 1, 2, 3, 4, 1, 2, 3, 4. The complex sequential movement was $1,2,4,3,4,2,1,3$. In both cases, 1 was the index Wnger, 2 the middle Wnger, 3 the ring Wnger, and 4 the little Wnger.

\section{Experimental procedure}

The subjects were examined in Wve conditions: (1) a simple movement observation condition; (2) a simple movement execution condition; (3) a complex movement observation condition; (4) a complex movement execution condition; and (5) a control condition.

Multichannel EEG was recorded while participants sat in a darkened room with their forearms pronated lying on armrests. To reduce eye movement and other electromyographic artifacts throughout the EEG data collection, subjects were asked to avoid blinking by $W$ xing their attention on a target point placed on a screen situated $1.30 \mathrm{~m}$ in front of them and to keep their jaw relaxed. These instructions were provided before each condition. A metronome, set at $2 \mathrm{~Hz}$, was used to regulate temporal consistency and similar experimental parameters in the control, observation, and execution conditions (Manganotti et al. 1998).

Before EEG data collection, the subject memorized and performed the simple and complex Wnger movements until she/he was able to complete them without making an error
Wve times in a row. This prevented assessment of a learning process during the EEG acquisition and ensured regular, stable performance throughout the experiment (Catalan et al. 1998).

\section{Movement observation condition}

Each subject performed 40 trials for the observation of the simple task and 40 trials for the observation of the complex task. Each trial comprised four stages which were shown to the subjects via a video display. Instructions were provided to the subjects requesting them to observe the movement with the goal of replicating it at a later stage.

During the Wrst stage of each trial $(t=0-4 \mathrm{~s})$ the subject watched an amber monitor screen. This screen warned the subject of the impending requirement to observe a video. During the second stage $(t=4-9 \mathrm{~s})$, subjects observed a video of a human model performing either a simple or a complex Wnger movement sequence at $2 \mathrm{~Hz}$. In the third stage ( $t=9-12 \mathrm{~s})$ a white background was presented requiring the subject to stay focused. In the fourth stage $(t=12$ $15 \mathrm{~s}$ ), a red monitor screen invited the subject to relax, to think of nothing, and to blink her/his eyes if necessary (see Fig. 1). The time interval between the beginning of viewing the movement and the onset of the next was $15 \mathrm{~s}$ (see Fig. 1). During the movement observation conditions, the experimenter controlled for Wnger movements; where movement was observed, the trial was discarded.

\section{Movement execution condition}

Each subject performed physically 40 trials of the simple movement and 40 trials of the complex movement following a similar procedure to the observation trials. The Wrst stage warned the subject about the imminent movement requirement. In the second, a black background was presented to the subject. During this stage, she/he performed the simple or complex Wnger movement at $2 \mathrm{~Hz}$. In the third stage, a white background invited the subject to remain focused. In the fourth stage, a red background prompted the

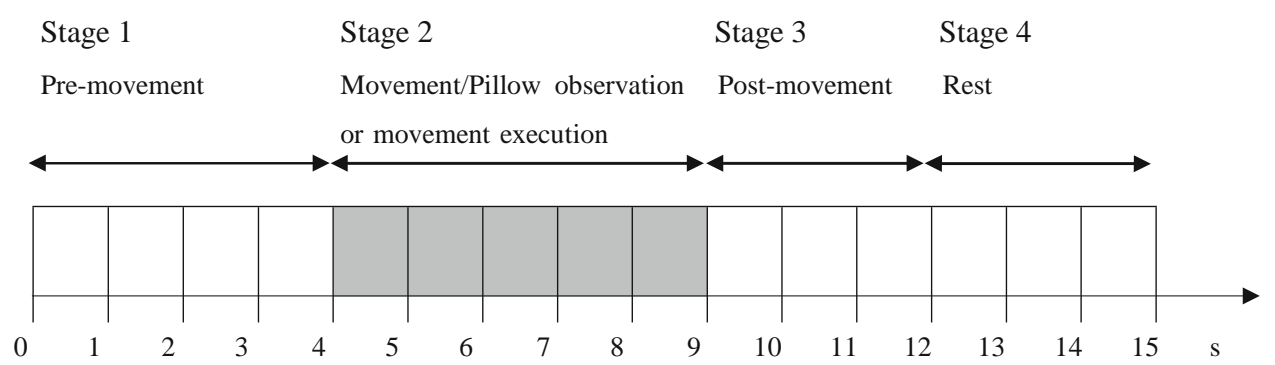

Fig. 1 Schema for one trial to be analyzed oV-line irrespective of condition. Shaded sections were used for EEG analysis. Stage 2 lasted $5 \mathrm{~s}$ since two periods of $500 \mathrm{~ms}$ (i.e., $500 \mathrm{~ms}$ before the onset of the movement and $500 \mathrm{~s}$ after its oVset/end) were added to the $4 \mathrm{~s}$ duration of the

movement. During these time lapses, the subject could see the hand and Wngers at rest. This was done to allow the subject to understand the context in which the movement was performed and to avoid abrupt video image changes 
subject to stop the movement, to relax, and to blink her/his eyes if required. The time interval between the beginning of one movement and the onset of the next was $15 \mathrm{~s}$ (see Fig. 1). During the movement execution condition, the experimenter checked the accuracy of the movements performed by the subjects; only correct series of Wnger-thumb contacts were retained for analysis. Where a subject made more than $\mathrm{W}_{\mathrm{ve}}$ mistakes while performing the movement, the data was discarded.

\section{Control condition}

Each subject performed 40 trials observing a pillow. Each trial was conducted similar to those used in the movement observation condition. However, instead of observing a movement, the participant observed a pillow for $5 \mathrm{~s}$ (see Fig. 1). Observing a stationary, non-biological object for the control condition was adopted based on the Wndings of Grafton et al. (1996). They reported that observation of movement was better contrasted with observation of an object than observation of a static hand since this can imply movement more than the inert object.

All trials for each of the $W$ ve conditions were triggered using a speciWcally designed interface based on a photoresistive diode which responded to the screen color change. Five, 10-min blocks of 40 trials were performed. Each block was separated by a 5-min rest period. Irrespective of the movement complexity, the movement observation was performed Wrst and was followed by the movement execution condition. The ordering was the same for the eleven subjects since they were required to observe the movement and replicate it at a later stage in order to investigate the role of mirror neurons in imitation (viz., Rizzolatti and Craighero 2004). The $W_{v e}$ conditions were structured into three main conditions: (1) The simple movement observation-execution conditions; (2) the complex movement observation-execution conditions; and (3) the control condition. The order of the three main conditions was counterbalanced across subjects.

\section{Data acquisition and recording}

Electrical brain activity was recorded from $19 \mathrm{Ag} / \mathrm{AgCl}$ pad electrodes held on the head with a rubber cap (Fp1, Fp2, Fz, F7, F8, F3, F4, Cz, C3, C4, PZ, P3, P4, T3, T4, T5, T6, O1, and $\mathrm{O} 2$ ) and placed in accordance with the international 10-20 system (Jasper 1958). Mastoids were used for the reference electrodes and the ground electrode was located on the forehead. Electro-oculograms (EOG) were also registered from the canthi of both eyes (horizontal EOG) and the supra and infra orbital of the right eye (vertical EOG). Electrode impedance was kept homogenously below $5 \mathrm{kQ}$ throughout the experimentation and was checked systemat- ically between the two blocks of trials. AmpliWer bandwidth was set between 0.15 and $114 \mathrm{~Hz}$ using a computerbased EEG recorder (Coherence, Deltamed, Paris, France). Baseline-corrected activity was sampled at $256 \mathrm{~Hz}$. AD resolution was 16 bit.

\section{Synchronization likelihood}

Synchronization likelihood is a measure of generalized synchronization between two time series. It is sensitive to linear as well as nonlinear coupling, and suitable for nonstationary data. SL is the likelihood that recurrence of a pattern in time series $\mathrm{X}$ at two times. I and $\mathrm{J}$ will coincide with recurrence of patterns in time series $\mathrm{Y}$ at the same times I and $\mathrm{J}$. The patterns are deWned in terms of state-space vectors obtained by time-delay embedding of the data. The SL takes on values between $P_{\text {ref }}$ (no coupling) and 1 (complete coupling). $P_{\text {ref }}$ is a parameter of the computation of synchronization likelihood, and is usually chosen as $P_{\text {ref }}=0.01$. Technical details can be found in Stam and van Dijk (2002) and Montez et al. (2006).

\section{Data processing}

EEG data were analyzed in four frequency bands: $8-10$, $10-13,13-20$, and $20-30 \mathrm{~Hz}$ and data processing was composed of three steps: segmentation of EEG trials, removal of artifacts, and SL computation.

\section{Segmentation of EEG trials}

Forty trials were completed during each ten-minute condition. Each trial was subdivided into four stages. For all conditions, details of these stages are provided in the experimental procedure section. Only the movement stage (4-9 s) was used for the EEG analysis (see Fig. 1).

\section{Removal of artifacts}

The artifact reduction process comprised two stages. First, all movement stage trials were visually inspected and trials

Table 1 Summary of the 5 (conditions) $£ 9$ (electrode sites) ANOVAs for each frequency band and for the movement stage

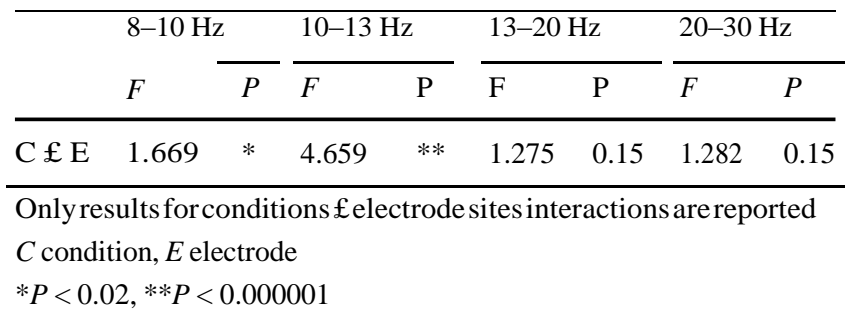


with ocular artifacts were corrected via the Semlitsch et al.'s (1986) method. The principle of this method being to subtract a fraction of an electro-oculogram (EOG) from the EEG. More speciWcally, a regression analysis associated with artifact averaging is used. This artifact removal was performed with Neuroscan 4.1 software (Revision A, 1999). The following options were selected: trigger, positive direction; threshold, 20\%; blink values, two minimum sweeps; average artifact duration, $400 \mathrm{~ms}$, and VEOG as the channel used in this computation; review, maxima and blinks enabled; and output, LDR + CNT. Second, for all the movement stage trials, slow drifts were removed by linear trend corrections (linear detrend module of Neuroscan 4.1 software).

\section{Synchronization likelihood computation}

Synchronization likelihood (SL) was computed for the movement stage for each of the trials of the $\mathrm{W}_{\mathrm{ve}}$ conditions, for each subject, frequency band, and electrode site. The SL values of a particular condition were averaged for each subject, frequency band, and electrode site. Parameters for the computation of the SL were: 1 sample for the lag, 10 for the embedding dimension, 10 for the Theiler correction $\left(w_{1}\right) ; 1$ for $w_{2} ; 0.010$ for $P_{\text {ref }}$; and 1 for the speed. These parameter choices were necessary in view of the very short duration of the movement stage to which the synchronization likelihood was applied.

\section{Statistical analysis}

All statistical analyses were performed using Statistica 7.1. From the electrode montage employed, F4, F3, P4, P3, FZ, and PZ were chosen for analysis because: (1) in humans, many frontal and parietal areas have been shown to possess mirror properties (Rizzolatti et al. 2002), and (2) the agranular frontal cortex (i.e., motor cortex) and the parietal lobe form the basic components of the motor system (Rizzolatti et al. 1998, 2002). CZ was selected because it overlies the supplementary motor area which is known to be involved in the generation of complex movement and planning of temporally sequential movement (Shibasaki et al. 1993). C4 and $\mathrm{C} 3$ were also chosen because oscillations from sites located over sensorimotor areas are thought to reXect the MN system activity (Pineda 2005). SL for these nine electrode sites was considered.

For each of the four frequency bands, 5 (conditions) $£ 9$ (electrode sites) repeated-measures ANOVAs were completed. There were two within-subject factors; condition (Wve levels: control, simple movement observation, complex movement observation, simple movement execution, and complex movement execution) and electrode site (nine levels corresponding to the nine EEG channels). Post hoc comparisons were calculated using Fisher's LSD test where ANOVA results were signiWcant. Before the ANOVA computations, the normality of the data was checked with the Kolmogorov-Smirnov test. To verify the sphericity assumption in repeated measures designs, the Mauchley's sphericity test was used.

These ANOVAs were computed to determine whether: (1) control condition SL values were signiW cantly diVerent from observation and execution conditions SL values, (2) simple movement SL values were signiWcantly diVerent from complex movement SL values under the observation and execution conditions, and (3) SL values during observation were signiWcantly diVerent from those during actual execution.

\section{Results}

Behavioral results

The percentages of correct Wnger taps performed by the subjects were $87 \%$ for the complex movement (13\% of trials were removed from analysis) and $100 \%$ for the simple movement. No subjects were excluded from the study. During the observation condition, subjects did not move their Wngers and no trials were removed from the subsequent analysis.

\section{SL for electrode sites}

Four 5 (conditions) $£ 9$ (electrode sites) ANOVAs were computed. The EEG data were normally distributed and no sphericity violations were observed. Only results for conditions by electrode sites interactions have been reported here since they were directly linked to the goals of the present study (see Table 1).

No signiWcant conditions $\mathfrak{E}$ electrode sites interactions were found for the $13-20$ and $20-30 \mathrm{~Hz}$ frequency bands (see Table 1). However, signiWcant conditions $£$ electrode sites interactions were observed in the movement stage for: (1) the $8-10 \mathrm{~Hz}$ band, $F(32,320)=1.669, P<0.02$; (2) the $10-13 \mathrm{~Hz}$ band, $F(32,320)=4.659, P<0.000001$ (see Table 1). Fisher's LSD tests were computed and three results were found.

First, during the movement stage, for the $8-10$ and 10 $13 \mathrm{~Hz}$ frequency bands, Fisher's LSD post hoc test analysis revealed signiWcant diVerences between the simple observation condition and the control condition for all the electrode sites of interest except for P4 in 10-13 Hz. SigniWcant diVerences were also found between the complex observation condition and the control condition in the two frequency bands except for F4 and P3 in $8-10 \mathrm{~Hz}$ and P4 in $10-13 \mathrm{~Hz}$ (see Fig. 2). Besides, signiWcant diVerences were 


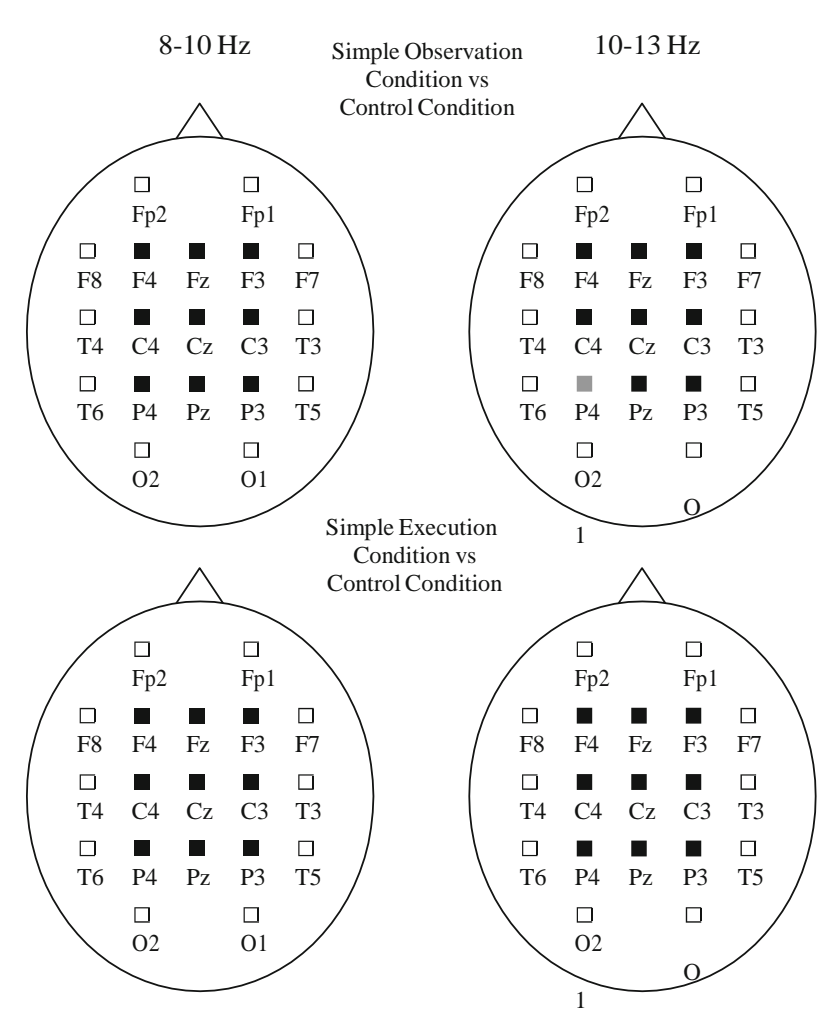

Fig. 2 Schemas indicating the scalp locations. Black and gray squares indicate the positions of electrodes of interest. SigniWcant diVerences in synchronization likelihood values between: a the simple observation condition and the control condition, $\mathbf{b}$ the complex observation condi-

detected in each of the two frequency bands between the execution conditions and the control condition for all the nine electrode sites of interest (see Fig. 2). Synchronization values were lower in the control condition compared to the observation and execution conditions.

Second, during the movement stage, for the 8-10 and $10-13 \mathrm{~Hz}$ frequency bands, and electrode sites of interest, synchronization values during the simple observation condition were not signiW cantly diVerent from the synchronization values during the complex observation condition. Similar results were found for the execution condition except for $\mathrm{C} 3$ and $\mathrm{FZ}$ in $10-13 \mathrm{~Hz}$. In these two cases, synchronization values were lower in the simple execution condition compared to the complex execution condition ( 0.083 vs. $0.088, P=0.038$ for C3; 0.090 vs. 0.095, $P=0.027$ for $\mathrm{FZ}$ ).

Third, Fisher's LSD post hoc test analysis showed signiWcant diVerences during the movement stage under the conditions of simple observation and simple execution for the $8-10 \mathrm{~Hz}$ band for F4, F3, C4, C3, P3, FZ, and CZ. For the $10-13 \mathrm{~Hz}$ band, signiW cant diVerences between simple observation and simple execution were also found for F4, F3, C4, C3, FZ, and CZ (see Figs. 3, 4). Similar results were found for complex observation and complex execution

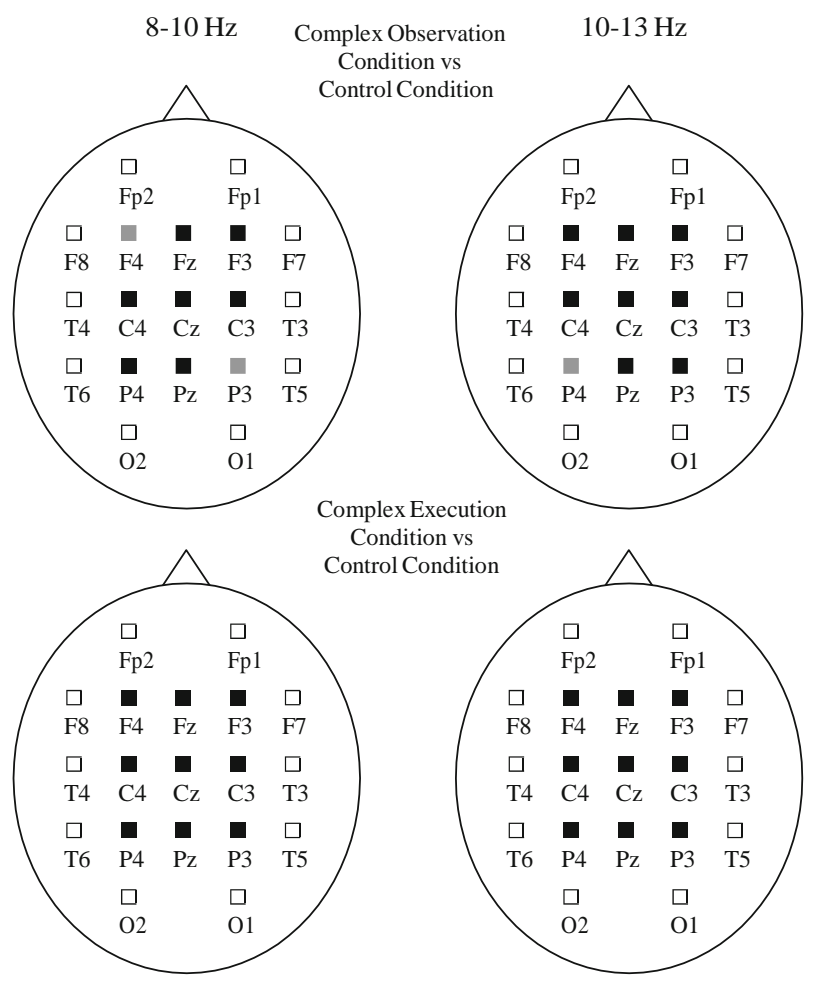

tion and the control condition, $\mathbf{c}$ the simple execution condition and the control condition, and $\mathbf{d}$ the complex execution condition and the control condition are represented by black squares $(P<0.05)$. Non signiWcant diVerences are represented by gray squares $(P>0.05)$

conditions for the $8-10$ and $10-13 \mathrm{~Hz}$ frequency bands. In both frequency bands, SL during execution was higher than during observation (see Figs. 3, 4). These Wndings show that for the $8-10$ and $10-13 \mathrm{~Hz}$ frequency bands, the observation-execution comparisons between the simple movement condition and the complex movement condition displayed similar synchronization patterns: SigniWcant and non signiWcant diVerences in SL were found at the same electrode sites (see Figs. 3, 4). More speciWcally, although there were some exceptions for parietal sites, the main Wnding was one of no signiWcant diVerences in SL for electrodes located in the parietal cortex (i.e., P4, PZ, P3) (see Figs. 3, 4).

\section{Discussion}

The primary aim of this study was to examine EEG synchronization during the physical execution and observation of simple and complex sequential Wnger movements. EEG functional connectivity was analyzed for the $8-30 \mathrm{~Hz}$ frequency band using a general measure, SL, sensitive to linear and non-linear coupling. The discussion has been organized into three sections. The Wrst discusses methodological considerations. The second considers task complexity, 

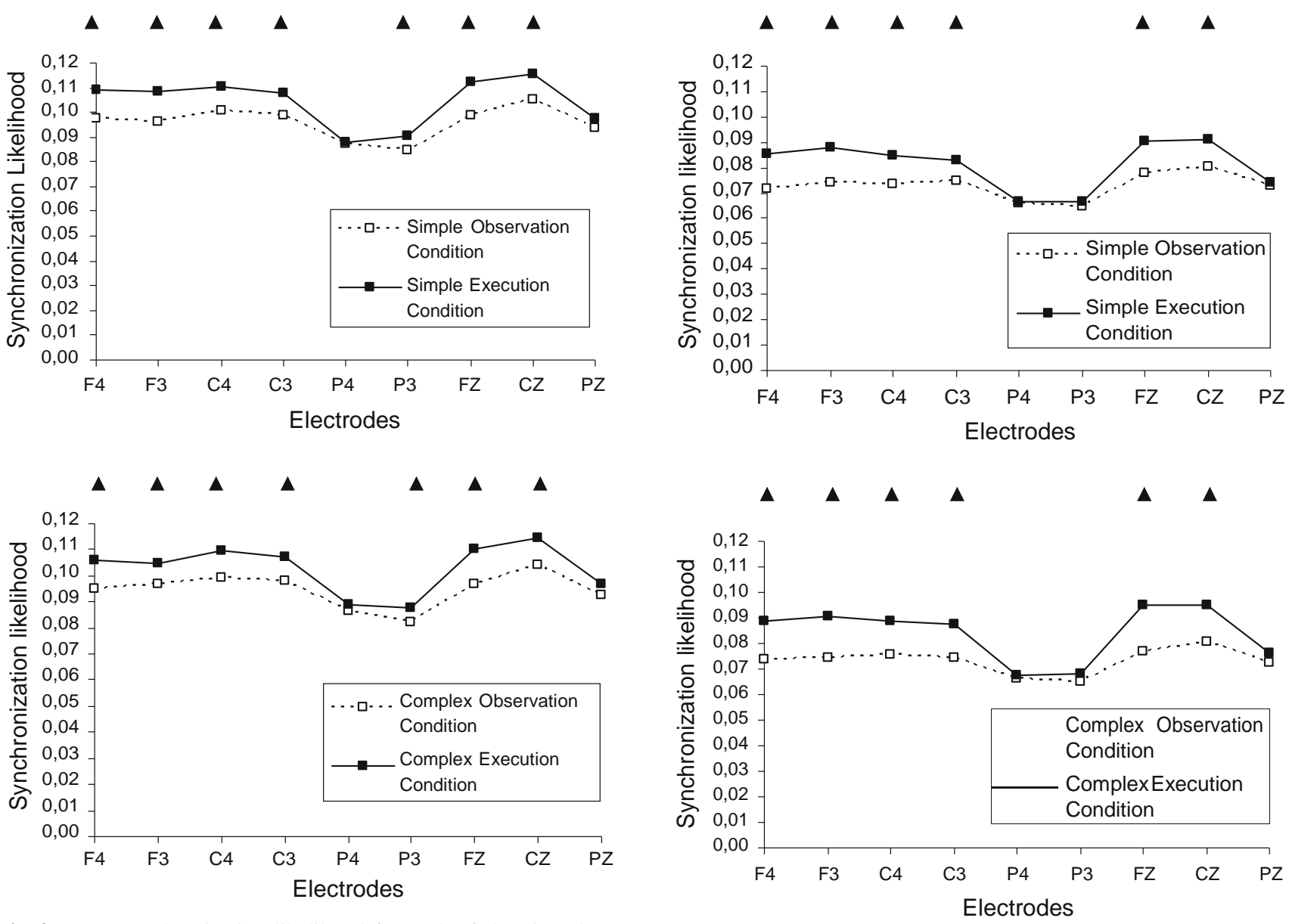

Fig. 3 Mean synchronization likelihood for each of the nine electrodes in the conditions of observation and execution in the $8-10 \mathrm{~Hz}$ frequency band during the movement stage. Filled triangle, $P$ is considered signiWcant

and the third reviews the existence of a human MN system. Our interpretation of the results has been made cautiously, since a lack of signiWcant diVerence in EEG activity between two experimental conditions does not automatically imply equality. Activity diVerences may exist but EEG as a technique may be unable to detect the diVerences which may be related to deeper motor structures (e.g., basal ganglia) whose activity is not present at the scalp.

\section{Methodological considerations}

Cortical synchronization during the resting state (i.e., during the control condition) for the nine electrode sites of interest was used as the control. Higher SL values were found during the execution and observation conditions in comparison to those obtained during the control condition for electrodes located in the fronto-centro-parietal cortex in the $8-10$ and $10-13 \mathrm{~Hz}$ frequency bands. However, no signiWcant diVerences were detected between the observation and execution conditions and the control condition for the

Fig. 4 Mean synchronization likelihood for each of the nine electrodes in the conditions of observation and execution in the $10-13 \mathrm{~Hz}$ frequency band during the movement stage. Filled triangle, $P$ is considered signiWcant

$13-20$ and $20-30 \mathrm{~Hz}$ frequency bands. An explanation for the results found in these higher bands is not clear.

One reason may be attributed to the oscillations in diVerent frequency bands that subserve diVerent functions (Chen et al. 2003; GerloV et al. 1998). The $8-10 \mathrm{~Hz}$ band is known to be related to attentional processes, whereas the $10-13 \mathrm{~Hz}$ band is related to semantic long-term memory processes (Klimesch 1996, 1999). In contrast, the 13-20 and 20$30 \mathrm{~Hz}$ frequency bands are closely associated with motor learning, motor preparation (Alegre et al. 2004), attentional mechanisms (Chen et al. 2003) and more cognitive tasks such as face recognition (Ozgoren et al. 2005). Therefore, it could be suggested that the $8-10$ and $10-13 \mathrm{~Hz}$ frequencies may reXect aspects of information processing which are distinguishable from those occurring during the control condition, whereas frequencies within the $13-30 \mathrm{~Hz}$ range do not. A second reason could be related to the choice of the control condition used for the baseline. It seems an ideal baseline condition does not exist. Wicker et al. (2003) have also stressed the problem of choosing an appropriate baseline for 
cognitive tasks involving integrated brain areas and Morcom and Fletcher (2007) have stated that "Any control state, no matter how carefully it is selected, is just another task state with its own unique areas of activation" (pp. 1074). Indeed, baseline values will show variation both within and across participants; therefore, a resting state is far from being an inactive state (Binder et al. 1999; Buzsaki 2006). When an individual is not actively committed to a behavior, mental activities can occur spontaneously, such as day dreams (e.g., Stark and Squire 2001), free association (e.g., Mazoyer et al. 2001), autobiographic episodes (e.g., Mazoyer et al. 2001), and inner speech and imagery (e.g., Mazoyer et al. 2001). Some researchers have demonstrated that, during a resting state, interactions between brain areas can be more extensive than during the completion of diversiWed transitive actions (e.g., Mazoyer et al. 2001; Shulman et al. 1997; Wicker et al. 2003).

In the literature, four possible conditions have been considered. First, in EEG/MEG studies, baseline periods are measured a few seconds prior to the event of interest (Pfurtscheller and Lopes da Silva 1999). Second, when the experimental task is performed with the eyes closed, researchers have designed rest conditions with the eyes closed also (e.g., Stam et al. 2002a, b). Third, baseline conditions have consisted of observing a static picture of a hand (e.g., Jonas et al. 2007). Finally, baselines have comprised viewing a Wxation cross or a monochrome background without a hand stimulus (e.g., Muthukumaraswamy and Johnson 2004). In the present study, the Wrst option was not chosen because of the speciWcity of the experimental procedure. The period before the onset of the task was a preparation stage for the impending task. The second option was also not selected because the experimental tasks required the subjects to have their eyes open. The third was not appropriate because the exposure to a static hand stimulus may have reduced the responsiveness of the mirror neuron system (Jonas et al. 2007). Recently, it has been shown that viewing static body postures suggesting a transition to action may activate the MN system (Urgesi et al. 2006). The problem, however, is knowing whether the static body stimulus is perceived as containing implied motion or not. Therefore, the fourth option seemed to be the most appropriate since cortical activations were clearer when action observation was compared to object observation rather than a static hand (Grafton et al. 1996).

\section{Complexity}

Contrary to our hypothesis, complex movements, compared to simple movements, did not generate greater interregional synchronization under the observation and execution conditions in the four frequency bands with the exception of $\mathrm{C} 3$ and $\mathrm{F} 3$ in the $10-13 \mathrm{~Hz}$ frequency band under the execution condition. Apart from these two cases, during the movement stage, and for all other frequency bands, no signiWcant diVerences in SL were found between simple and complex movements irrespective of condition. This Wnding may be explained by a number of reasons. First, the lack of synchronization diVerence between the simple and complex execution conditions in the present study may be due to a saturation or ceiling eVect. This eVect has been observed by Sadato et al. (1996b) with positron emission tomography (PET). They showed that regional cerebral blood Xow (rCBF) increased in the primary sensorimotor cortex (SM1) with Wnger movement frequencies up to $2 \mathrm{~Hz}$ and tended to a plateau at higher frequencies. Though Sadato et al. (1996b) have examined activation and not synchronization, it may be possible that a similar ceiling eVect occurred in EEG at $2 \mathrm{~Hz}$ as has been suggested by Manganotti et al. (1998). Synchronization values may have reached a steady-state and synchronization measurements between simple and complex movement could not, therefore, be diVerentiated. Second, it is possible that SL is not an appropriate marker to detect diVerences in the synchronization of distant neuronal populations between simple and complex motor acts. However, Micheloyannis et al. $(2003,2005)$ have shown that synchronization likelihood was able to discriminate cognitive tasks at diVerent complexities, so this oVer seems unlikely. Third, it may be possible that what was deWned as the simple sequential movement may actually be a complex sequential movement since the moveable hand was outside of the subject's visual array. Since the task required the touching of the tip of the thumb with the tips of the four Wngers, with no visual guidance, it may have been perceived as more complex than Wrst thought. Through anecdotal debriefs, some subjects reported being concerned about missing the contact between the thumb and the other four Wngers. Therefore, it may be that participants observed and executed two complex sequential movements. Finally, it may be possible that, because of the pre-experimental training session, the two movements could not be diVerentiated by level of diYculty since they both became overlearned and were performed quasi-automatically. The subjects may have reached an automatic stage of learning for each movement; whether the subjects had reached an automatic stage is debatable. Automaticity is used "to indicate that a person performs a skill or engages in an informationprocessing activity without demands on attention capacity" (Magill 2004, pp. 151). Assessment of automaticity has been shown through dual task paradigms where minimum interference should be observed on the primary task to conclude that it had reached automaticity (Passingham 1996). For example, Wu et al. (2004) developed a dual task paradigm with a Wnger movement and a letter-counting task for the interference task. 
The results of the present study also showed for all frequency bands that similar synchronization patterns under the simple and complex movement conditions were found when observation and execution conditions were compared. This strengthens the proposition that the MN system may not be sensitive to motor acts which have diVerent degrees of complexity or, as mentioned above, that the simple experimental task may have been perceived as more complex than Wrst thought.

The present study does not allow us to support claims for one reason over another one. Additional investigations will be necessary to resolve this issue.

\section{Existence of a mirror neuron system}

Consistent with our prediction, the data tend to provide evidence for an EEG equivalence between the observation and execution conditions; this was true for the 13-20 and 20-30 Hz frequency bands. For the two movements, no signiWcant diVerences in SL were found between the observation and execution conditions during the movement stage for the nine electrode sites of interest. These Wndings suggest the validity of the mirror neuron system activity through the 13-20 and 20-30 Hz frequency bands, as usually deWned by Rizzolatti and Craighero (2004), since similar or, more correctly, non-diVerent, cortical oscillations for an action, either observed or executed, were generated. Further, interpretation of these results should be made cautiously as the lack of any statistical signiWcance between the control condition and the observation or execution conditions is of concern. However, in the present study, there were diVerences, albeit not statistically signiW cant, between SL values in the control condition and those in the other conditions. In these cases, control SL values were lower than observation and execution SL values.

However, in the $8-10$ and $10-13 \mathrm{~Hz}$ frequency bands, SL data revealed that congruence between the observation and execution conditions was not complete. SigniWcant diVerences across the observation and execution conditions were observed for the Wnger movements and for all the electrodes of interest except for some located in the parietal cortex. The pattern of synchronization under the observation condition was similar but weaker in intensity to that recorded when subjects perform the observed action. The fact that, the areas of interest, known to be involved in motor control, display patterns of synchronization during observation of an action akin to those revealed during the execution of the same action can be reported as an indirect evidence for a human MN system. This viewpoint is supported by the $\mathrm{MN}$ research Wndings of a number of groups (Fadiga et al. 1995; Iacoboni et al. 1999, 2001; Koski et al. 2002, 2003; Muthukumaraswamy and Johnson 2004;
Muthukumaraswamy et al. 2004; Patuzzo et al. 2003). These authors have shown, with a variety of diVerent methods, that observation of intransitive, non-object-related actions, can activate the human MN system. More speciWcally, Muthukumaraswamy et al. (2004) reported that the human MN system was active during the observation of transitive and intransitive actions and that the magnitude of the mu rhythm was related to the characteristics of the observed action. Desynchronization of the mu rhythm was greater during the observation of a manipulandum grip than during the observation of an empty grip (Muthukumaraswamy et al. 2004). The proposal from these Wndings was that the cortical representations of motoric actions, directed towards objects, are either diVerent or more salient than those of non-object directed actions.

We also feel it is important to oVer an explanation for the lack of signiWcance between the observation and execution condition in the $8-10$ and $10-13 \mathrm{~Hz}$ frequency bands for some of the parietal electrode sites. Apart from some results to the contrary (e.g., Iacoboni et al. 1999), execution, in contrast to observation mainly involves fronto-central areas as recognized by Rao et al. (1993), Shibasaki et al. (1993), and Sadato et al. (1996a). This may explain why synchronization likelihood (SL) values for parietal electrode sites are lower than the SL values of electrodes located in the central and frontal areas. The parietal SL values collected during the execution condition are very close to parietal SL values recorded during the observation condition since it is known that parietal areas play an important role in the observation process (Iacoboni et al. 1999, Rizzolatti et al. 2002).

Finally, taken all together, the results in frequency bands lying with a range of $8-30 \mathrm{~Hz}$ seem to provide additional, albeit indirect, evidence for $\mathrm{MN}$ activity associated with intransitive tasks.

\section{Conclusions}

The use of the non-linear SL technique (Stam and van Dijk 2002) allowed for detailed consideration of some of the mechanisms of functional integration in observation and execution conditions. The results indicated that cortical connectivity did not seem to be sensitive to motor acts of diVerent levels of complexity under the observation and execution conditions. They also provided additional, indirect evidence, for human $\mathrm{MN}$ activity for intransitive tasks.

Acknowledgments This study was supported by a grant from the French Ministry of Health, Youth, and Sports. The authors are grateful to the participants who participated in the study. They would also like to thank M. Paisley for the video footage editing and the two anonymous reviewers for their helpful suggestions and comments. This study is dedicated to Gui Calmels. 


\section{References}

Alegre M, Garcia de Gurtubay I, Labarga A, Iriatre J, Malanda A, Artieda J (2004) Alpha and beta oscillatory activity during a sequence of two movements. Clin Neurophysiol 115:124-130

Binder JR, Frost JA, Hammeke TA, Bellgowan PSF, Rao SM, Cox RW (1999) Conceptual processing during the conscious resting state: a functional MRI study. J Cogn Neurosci 11:80-93

Buccino G, Binkofski F, Fink GR, Fadiga L, Fogassi L, Gallese V, Seitz RJ, Zilles K, Rizzolatti G, Freund HJ (2001) Action observation activates premotor and parietal areas in a somatotopic manner: an fMRI study. Eur J Neurosci 13:400-404

Buzsaki G (2006) Rhythms of the brain. Oxford University Press, New York

Catalan MJ, Honda M, Weeks RA, Cohen LG, Hallett M (1998) The functional neuroanatomy of simple and sequential Wnger movements: a PET study. Brain 121:253-264

Chen R, GerloV C, Hallett M, Cohen LG (1997) Involvement of the ipsilateral motor cortex in Wnger movements of diVerent complexities. Ann Neurol 41:247-254

Chen Y, Ding M, Kelso JAS (2003) Task-related power and coherence changes in neuromagnetic activity during visuomotor coordination. Exp Brain Res 148:105-116

Cochin S, Bathelemy C, Lejeune B, Roux S, Martineau J (1998) Perception of motion and qEEG activity in human adults. Electroencephalogr Clin Neurophysiol 107:287-295

Cochin S, Barthelemy C, Roux S, Martineau J (1999) Observation and execution of movement: similarities demonstrated by quantiWed electroencephalography. Eur J Neurosci 11:1839-1842

Dassonville P, Lewis SM, Zhu XH, Ugurbil K, Kim SG, Ashe J (1998) EVects of movement predictability on cortical motor activation. Neurosci Res 32:65-74

Dhamala M, Pagnoni G, Wiesenfeld K, Zink CF, Martin M, Berns GS (2003) Neural correlates of the complexity of rhythmic Wnger tapping. Neuroimage 20:918-926

Di Pellegrino G, Fadiga L, Fogassi L, Gallese V, Rizzolatti G (1992) Understanding motor events: a neurophysiological study. Exp Brain Res 91:176-180

Fadiga L, Fogassi L, Pavesi G, Rizzolatti G (1995) Motor facilitation during action observation: a magnetic stimulation study. J Neurophysiol 73:2608-2611

Friston KJ (2000) The labile brain. I. Neuronal transients and non linear coupling. Philos Trans R Soc Lond B Biol Sci 355:215-236

Gallese V, Fadiga L, Fogassi L, Rizzolatti G (1996) Action recognition in the premotor cortex. Brain 119:593-609

Gastaut HJ, Bert J (1954) EEG changes during cinematographic presentation. Electroencephalogr Clin Neurophysiol 6:433-444

GerloV C, Richard J, Hadley J, Schulman AE, Honda M, Hallett M (1998) Functional coupling and regional activation of human cortical motor areas during simple, internally paced and externally paced Wnger movements. Brain 121:1513-1531

Grafton ST, Arbib MA, Fadiga L, Rizzolatti G (1996) Localization of grasp representations in humans by positron emission tomography. 2. Observation compared with imagination. Exp Brain Res 112:103-111

Grèzes J, Armony JL, Rowe J, Passingham RE (2003) Activations related to "Mirror" and "Canonical" neurones in the human brain: a fMRI study. Neuroimage 18:928-937

Hummel F, Kirsammer R, GerloV C (2003) Ipsilateral cortical activation during Wnger sequences of increasing complexity: representation of movement diYculty or memory load? Clin Neurophysiol 114:605-613

Iacoboni M, Woods RP, Brass M, Bekkering H, Mazziotta JC (1999) Cortical mechanisms of human imitation. Science 286:25262528
Iacoboni M, Koski LM, Brass M, Bekkering H, Woods RP, Dubeau MC, Mazziotta JC, Rizzolatti G (2001) ReaVerent copies of imitated actions in the right superior temporal cortex. Proc Natl Acad Sci USA 98:13995-13999

Jackson PL, MeltzoV AN, Decety J (2006) Neural circuits involved in imitation and perspective-taking. Neuroimage 31:429-439

Jasper HH (1958) Report of the committee on methods of clinical examination in electroencephalography. Electroencephalogr Clin Neurophysiol 10:370-375

Jonas M, Siebner HR, Biermann-Ruben K, Kessler K, Baumer T, Buchel C, Schnitzler A, Munchau A (2007) Do simple intransitive Wnger movements consistently activate frontoparietal mirror neuron areas in humans? Neuroimage 36:T44-T53

Kitamura J, Shibasaki H, Takagi A, Nabeshima H, Yamaguchi A (1993) Enhanced negative slope of cortical potentials before sequential as compared with simultaneous extensions of two $\mathrm{Wn}$ gers. Electroencephalogr Clin Neurophysiol 86:176-182

Klimesch W (1996) Memory processes, brain oscillations and EEG synchronization. Int J Psychophysiol 24:61-100

Klimesch W (1999) Event-related band power changes and memory performance. In: Pfurtscheller G, Lopes da Silva FH (eds) Eventrelated desynchronization. Handbook of electroencehalography and clinical neurophysiology. Revised series, vol 6. Elsevier, New York, pp 161-178

Koski L, Wohlschlager A, Bekkering H, Woods RP, Dubeau MC, Mazziotta JC, Iacoboni M (2002) Modulation of motor and premotor activity during imitation of target-directed actions. Cereb Cortex 12:847-855

Koski L, Iacoboni M, Dubeau MC, Woods RP, Mazziotta JC (2003) Modulation of cortical activity during diVerent imitative behaviors. J Neurophysiol 89:460-471

Leistner S, Wubbeler G, Trahms L, Curio G, Mackert BM (2006) Tonic neuronal activation during simple and complex Wnger movements analyzed by DC-magnetoencephalography. Neurosci Lett 394:42-47

Lepage JF, Théoret H (2006) EEG evidence for the presence of an action observation-execution matching system in children. Eur $\mathrm{J}$ Neurosci 23:2505-2510

Magill RA (2004) Motor learning and control. Concepts and applications. McGraw-Hill, Boston

Manganotti P, GerloV C, Toro C, Katsuda H, Sadato N, Zhuang P, Leocani L, Hallett M (1998) Task-related coherence and task-related spectral power changes during sequential Wnger movements. Electroencephalogr Clin Neurophysiol 109:50-62

Mazoyer B, Zago L, Mellet E, Bricogne S, Etard O, Houdé O, Crivello F, Joliot M, Petit L, Tzourio-Mazoyer N (2001) Cortical networks for working memory and executive functions sustain the conscious resting state in man. Brain Res Bull $54: 287-298$

Micheloyannis S, Vourkas M, Bizas M, Simos PG, Stam CJ (2003) Changes in linear and nonlinear EEG measures as a function of task complexity: evidence for local and distant signal synchronization. Brain Topogr 15:239-247

Micheloyannis S, Sakkalis V, Vourkas M, Stam CJ, Simos PG (2005) Neural networks involved in mathematical thinking: evidence from linear and non-linear analysis of electroencephalographic activity. Neurosci Lett 373:212-217

Molnar-Szakacs I, Kaplan J, GreenWeld PM, Iacoboni M (2006) Observing complex action sequences: the role of the fronto-parietal mirror neuron system. Neuroimage 33:923-935

Montez T, Linkenkaer-Hansen K, van Dijk BW, Stam CJ (2006) Synchronization likelihood with explicit time-frequency priors. Neuroimage 33:1117-1125

Morcom AM, Fletcher PC (2007) Does the brain have a baseline? Why we should be resisting a rest. Neuroimage 37:1073-1082 
Muthukumaraswamy SD, Johnson BW (2004) Changes in rolandic mu rhythm during observation of a precision grip. Psychophysiology 41:152-156

Muthuswamy J, Thakor NV (1998) Spectral analysis methods for neurological signals. J Neurosci Methods 83:1-14

Muthukumaraswamy SD, Johnson BW, McNair NA (2004) Mu rhythm modulation during observation of an object-directed grasp. Cogn Brain Res 19:195-201

Oberman LM, McCleery JP, Ramachandran VS, Pineda JM (2007) EEG evidence for mirror neuron activity during the observation of human and robot actions: towards an analysis of the human qualities of interactive robots. Neurocomputing 70:2194-2203

OldWeld RC (1971) The assessment and analysis of handedness: the Edinburgh Inventory. Neuropsychologia 9:77-113

Ozgoren M, Basar-Eroglu C, Basar E (2005) Beta oscillations in face recognition. Int J Psychophysiol 55:51-59

Palus M (1996) Nonlinear in normal human EEG: cycles, temporal asymmetry, nonstationarity and randomness, not chaos. Biol Cybern 75:389-396

Passingham RE (1996) Attention to action. Philos Trans R Soc Lond B Biol Sci 351:1473-1479

Patuzzo S, Fiaschi A, Manganotti P (2003) Modulation of motor cortex excitability in the left hemisphere during action observation: a single- and paired-pulse transcranial magnetic stimulation study of self- and non-self-action observation. Neuropsychologia $41: 1272-1278$

Pfurtscheller G, Lopes da Silva FH (1999) Event-related. EEG/MEG synchronization and desynchronization: basic principles. Clin Neurophysiol 110:1842-1857

Pineda JA (2005) The functional signiWcance of mu rhythms: translating "seeing" and "hearing" into "doing". Brain Res Rev 50:57-68

Pulvermüller F, Mohr B (1996) The concept of transcortical cell assemblies: a key to the understanding of cortical lateralization and interhemispheric interaction. Neurosci Biobehav Rev 20:557-566

Pulvermuller F, Lutzenberger W, Preissl H, Birbaumer N (1995) Motor programming in both hemispheres: an EEG study of the human brain. Neurosci Lett 190:5-8

Rao SM, Binder JR, Bandettini PA, Hammeke TA, Yetkin FZ, Jesmanowicz A, Lisk LM, Morris GL, Mueller WM, Estkowski LD, Wong EC, Haughton VM, Hyde JS (1993) Functional magnetic resonance imaging of complex human movements. Neurology 43:2311-2318

Rizzolatti G, Craighero L (2004) The mirror-neuron system. Ann Rev Neurosci 27:169-192

Rizzolatti G, Carmada R, Fogassi L, Gentilucci M, Luppino G, Matelli M (1988) Functional organization of inferior area 6 in the macaque monkey: II. Area F5 and the control of distal movements. Exp Brain Res 71:491-507

Rizzolatti G, Luppino G, Matelli M (1998) The organization of the cortical motor system: new concepts. Electroencephalogr Clin Neurophysiol 106:283-296

Rizzolatti G, Craighero L, Fadiga L (2002) The mirror system in human. In: Stamenov MI, Gallese V (eds) Mirror neurons and the evolution of brain and language. John Benjamins Publishing Company, Philadelphia, pp 37-63
Sadato N, Campbell G, Ibanez V, Deiber MP, Hallett M (1996a) Complexity aVects regional cerebral blood Xow change during sequentialWnger movements. JNeurosci 16:2691-2700

Sadato N, Ibanez V, Deiber MP, Campbell G, Leonardo M, Hallett M (1996b) Frequency-dependent changes of regional cerebral blood Xow during Wnger movements. J Cereb Blood Flow Metab 16:2333

Semlitsch H, Anderer P, Schuster P, Presslich O (1986) A solution for reliable and valid reduction of ocular artifacts, applied to the P300 ERP.Psychophysiology 23:695-703

Shibasaki H, Sadato N, Lyshkow H, Yonekura Y, Honda M, Nagamine T, Suwazono S, Magata Y, Ikeda A, Miyazaki M (1993) Both primary motor cortex and supplementary motor area play an important role in complex Wnger movement. Brain 116:1387-1398

Shulman GL, Fiez JA, Corbetta M, Buckner RL, Miezin F, Raichle ME, Petersen SE (1997) Common blood Xow changes across visual tasks: II. Decreases in cerebral cortex. J Cogn Neurosci 9:648-663

Solodkin A, Hlustik P, Noll DC, Small SL (2001) Lateralization of motor circuits and handedness during Wnger movements. Eur J Neurol 8:425-434

Stam CJ, van Dijk BW (2002) Synchronization likehood: an unbiased measure of generalized synchronization in multivariate data sets. Physica D 163:236-251

Stam CJ, Pijn JP, SuVczynski P, Lopes da Silva FH (1999) Dynamics of the human alpha rhythm: evidence for non-linearity? Clin Neurophysiol 110:1801-1813

Stam CJ, van Walsum AM, Micheloyannis S (2002a) Variability of EEG synchronization during a working memory task in healthy subjects. Int J Psychophysiol 46:53-66

Stam CJ, van Walsum AM, Pijnenburg YAL, Berendse HW, de Munck JC, Ph Scheltens, van Dijk BW (2002b) Generalized synchronization of MEG recordings in Alzheimer's disease: evidence for involvement of the gamma band. J Clin Neurophysiol 19:562574

Stark CE, Squire LR (2001) When zero is not zero: the problem of ambiguous baseline conditions in fMRI. Proc Natl Acad Sci USA 98:12760-12765

Thakor NV, Tong S (2004) Advances in quantitative electroencephalogram analysis methods. Annu Rev Biomed Eng 6:453-495

Ulloa ER, Pineda JA (2007) Recognition of point-light biological motion: mu rhythms and mirror neuron activity. Behav Brain Res 183:188-194

Urgesi C, Candidi M, Fabbro F, Romani M, Aglioti S (2006) Motor facilitation during action observation: topographic mapping of the target muscle and inXuence of the onlooker's posture. Eur J Neurosci 23:2522-2530

Verstynen T, Diedrichsen J, Albert N, Aparicio P, Ivry RB (2005) Ipsilateral motor cortex activity during unimanual hand movements relates to task complexity. J Neurophysiol 93:1209-1222

Wicker B, Ruby P, Royet JP, Fonlupt P (2003) A relation between rest and the self in the brain? Brain Res Rev 43:224-230

Wu T, Kansaku K, Hallett M (2004) How self-initiated memorized movements become automatic: a functional MRI study. J Neurophysiol 91:1690-1698 